\title{
Health effects of pollution of operating theatre environment with halothane during anaesthesia; a comparative study
}

\author{
Ariadurai JSA, Gunaratne A, Samaraweera L, DeAlwis NAVCT, Sellathurai N \\ Lady Ridgeway Hospital for Children, Borella, Colombo,, Sri Lanka \\ Correspondence: Dr. J.S.A.Ariyadurai \\ e-mail: j.sylvanthi@gmail.com
}

\begin{abstract}
Introduction: The possible adverse effects of long-term anaesthetic gas exposure on medical personnel working in operating theatres were analysed. The results of this study could be used to improve the gas scavenging techniques.
\end{abstract}

Methods: An analysis of data obtained from a questionnaire administered to 40 health care workers working in the theatre environment (exposed group) and another 40 working outside the theatre (unexposed group).

Results: Symptoms such as headache (30 subjects vs 5 subjects), dizziness (18 subjects vs no subjects), drowsiness ( 30 subjects vs no subjects), and irritability ( 5 subjects vs no subjects) were significantly higher among exposed compared to the unexposed group. The Chi-square analysis showed a $\mathrm{P}$ value less than 0.001 for the above symptoms.

Conclusions: There is a higher prevalence of symptoms related to nervous system among people exposed to theater environment. Scavenging anaesthetic gases becomes difficult when un-cuffed tubes and open circuit are widely used, such as in paediatric practice. This significantly exposes the theatre staff to anaesthetic gases. Alow flow closed breathing circuit along with a scavenging system should be used at all times unless there is a reason not to do so.

Keywords: Pollution, scavenging, theatre environment

\section{Introduction}

Pollution of theatre environment with anaesthetic gases and vapour may cause significant adverse effects on the theatre staff, in particular on anaesthetists. This may contribute to a variety of effects ranging from tiredness and lack of concentration after work to recurrent miscarriages and elevated liver enzymes.

Scavenging of anaesthetic gases is a standard procedure in modern theatre facilities. However, even in a modern unit scavenging becomes difficult when un-cuffed tubes and open circuits are used as in paediatric surgery.
The objective of this research project was to determine whether staff members of the operating theatre have symptoms related to long-term anaesthetic gas exposure, such as fatigue, headache, dizziness, nausea, drowsiness, irritability and lack of agility, compared to the staff working outside the theatre environment.

\section{Methods}

The exposed group consisted of 40 doctors and nurses working in the theatre complex of Teaching hospital, Karapitiya and another 40 doctors and nurses working in the Surgical Intensive Care Unit, 
Medical Intensive Care Unit, Paediatric Baby Unit or Neonatal Unit formed the unexposed group. For the exposed group, only the doctors and nurses who regularly worked for 6 to 8 hours a day in the operation theatre for more than one month were considered. Those who used alcohol more than 40 units per week and those who were on antidepressants or sedatives were excluded from the study. Data were collected using a structured data collection form given to the exposed and unexposed groups.

\section{Results}

The exposed group consisted on 15 males. Number of doctors was 34 and the rest (6) were nurses. Mean age of the group was $39 \mathrm{ys}$. There were 10 males in the unexposed group. Number of doctors in the unexposed groups was 10 while the rest (30) were nurses. Mean age of the group was 30ys (Table 1).

Of the 40 in the exposed group, 35 had worked for an average duration of 8 hours a day in the theatre environment. Thirty reported some adverse effects after work. The commonest was headache (30 subjects) and in 20 of them headache has lasted for 1 to 2 hours after work. However, of the 30 who complained of headache after work eight were known to suffer from migraine.

In the unexposed group consisted of 40 subjects, only five reported headache after work. None of them complained about any other adverse effects inquired (Table 2).

Table 1: Characteristics of the subjects in the exposed and unexposed groups

\begin{tabular}{lll}
\hline Variable & $\begin{array}{l}\text { Exposed } \\
\text { group }\end{array}$ & $\begin{array}{l}\text { Unexposed } \\
\text { group }\end{array}$ \\
\hline Mean age (ys) & 39 & 30 \\
Number of males & 15 & 10 \\
Number of doctors & 34 & 10 \\
Number of nurses & 6 & 30 \\
\hline
\end{tabular}

Table 2: Preval ence of adverse effects among exposed and unexposed

\begin{tabular}{llll}
\hline Variable & $\begin{array}{l}\text { Exposed } \\
\text { group }\end{array}$ & $\begin{array}{l}\text { Unexposed } \\
\text { group }\end{array}$ & P value \\
\hline Headache & $30 / 40$ & $5 / 40$ & $<0.001$ \\
Dizziness & $18 / 40$ & $0 / 40$ & $<0.001$ \\
Drowsiness & $30 / 40$ & $0 / 40$ & $<0.001$ \\
Irritability & $5 / 40$ & $0 / 40$ & 0.021 \\
Nausea & 0 & 0 & - \\
Fatigue & 0 & 0 & - \\
\hline
\end{tabular}

P compares the proportions between the two groups

The P values for the symptoms of headache, dizziness, and drowsiness were statistically significant. 


\section{Discussion}

This study found a higher prevalence of symptoms related to nervous system among health care workers exposed to theater environment. Health care workers who also worked in a similar physical environment but not exposed to theater had a lower prevalence of these symptoms.

Carl Johan et al reported to the Scandinavian Journal of Work Environment the possible teratogenic effect of anaesthetic agents as shown by animal experiments. They also found a high incidence of pregnancy complications among female anaesthesiologists (1). The authors also observed that the method used for administering anaesthesia affects the exposure of the medical personnel involved. A face mask produced heavier exposure than administration via a tracheal tube. No rebreathing anaesthetic systems resulted in greater exposure. Also the exposure tended to be greater when the patient breathed spontaneously than when his respiration was controlled. The elimination of halothane was considerably lower. Low residual concentrations of anaesthetic agents were demonstrable in end-expired air for a long time in the studied group of theatre nurses (2).

Murrin has observed sporadic reports of varying illhealth (nausea, myalgia and headache) among personnel working in the theatre environment. By a process of elimination, he concludes that atmospheric pollution with volatile agents to be the most possible explanation for the reported ill-health (3). No one has yet postulated a safe limit of contamination of anaesthetic agents in the theatre environment. Murrin suggested that threshold limits are of little value because of the chronicity of exposure involved with theatre pollution (3). According to him considerable concentration occurs around the face mask through ill-fitting joints as well as diffusion through rubber. Contamination can also occur when patients are transferred from the anaesthetic room to the recovery (3).
According to many authors efficient venting of anaesthetic gases right at the source of leakage, generally results in very significant reduction in the concentration of air borne anaesthetics $(2,4-7)$.

Scavenging anaesthetic gases becomes difficult when un-cuffed tubes and open circuit are widely used, such as in paediatric practice. This significantly exposes the theatre staff to anaesthetic gases. A low flow closed breathing circuit along with the scavenging system should be used at all times unless there is a reason not to do so.

\section{References}

1. Carl Johan Gothe, Per Ovrum, Borje Hallen. Exposure to anaesthetic gases and ethanol during work in operating rooms, Scandinavian Journal of Work Environment \& Health, 1976; 2: 96-106.

2. Corbett TH, and Ball GL. Chronic exposure to methoxyflurane: A possible occupational hazard to anaesthesiologists. Anaesthesiology 1971; 34: 532-7.

3. Murrin KR. Atmospheric pollution with halothane in operating theatres A clinical study using activated charcoal. Anaesthesia, 1975;30: 12-7.

4. Askrog V and Petersen R. Contamination of operating theatres from inhalation anaesthetics and ionization radiation. Nord Med 1970; 83: 501-4.

5. Bruce DL. A simple way to vent anaesthetic gases. Anesth Analg (Cleve) 1973; 52: 595-8.

6. Pfaffli P, Nikki P, Ahlman K. Halothane and nitrous oxide in end-tidal air and venous blood of surgical personnel. Ann ClinRes 1972; 4: 273-7.

7. Whitcher CE, Cohen EN, Trudell JR. Chronic exposure to anaesthetic gases in the operating room. Anaesthesiology 1971; 35: 348-53. 